\title{
Analysis of the Third Movement of Beethoven Piano Sonata Op.13, Pathetique
}

\author{
Yingjie Cheng \\ Hubei Engineering University, Xiaogan Hubei, 432000, China
}

Keywords: Beethoven, The third movement of Pathetique, Harmonic analysis, Musical form analysis.

\begin{abstract}
The Sonata Pathetique is the summit of Beethoven piano Sonata at early stage, which is familiar by people due to the third dramatic and melodic movement. In this article, we reveal the features that the composer not only followed the classicism, but also showed the romance at that time through the overall and meticulous harmonic analysis of this movement, and clarify that the structure of this Sonata reflects both the features of rondo form and the principle of three-part structure and the structure of uniform Sonata form with middle tonality by the musical structure analysis. This Sonata can be called as creative model of piano Sonata.
\end{abstract}

\section{Introduction}

Beethoven is one of the famous German musicians and one of the representatives of Viennese Classical School. His music influences the development of world music profoundly, so he is honored as "master musician" by later generations. His 32 piano Sonatas are known as the models of piano music in the world music treasure-house ${ }^{[1]}$. The Sonata, Pathetique, was a gift created by Beethoven to his patron and admirer, Prince Lichnovsky, which was created at the beginning of 1798 and is the summit of Beethoven piano Sonata at early stage, and which also is familiar by people due to the third dramatic and melodic movement.

\section{Harmonic analysis}

\section{Exposition}

There are 8 bars in the theme of principal part with c minor starting from p, and the first half harmony of the theme of principal part is alternated on the main continuant from dominant to subdominant. The bars from 3 to 4 are half cadence with the progression of $\mathrm{D}_{6}-\mathrm{tsvi}_{7}-\mathrm{D}$, and tsvi takes the place of $\mathrm{t}_{4}{ }^{6}$ to play the role of passing. The bar 6 in the theme is from the dominant six chord to heavy-leading sixth-five chord, and next, it enters into the cadence $\mathrm{K}_{4}{ }^{6}-\mathrm{D}$-t of this phrase. The theme of principal part seems to be terminated perfectly, but its basso is a p rhythm and the primary chord is resolved in the basso part to weaken the perfect sense of cadence so that the music has the power to go on. The first half of the second phrase also progresses in the cadence of $\mathrm{K}_{4}{ }^{6}$-D-t, but the primary chord of cadence is short, and then $\mathrm{D}_{7} / \mathrm{s}$-s appears immediately and the music is modulated to subordinate direction. From bar 13 to 14 , the harmony configuration $\mathrm{D}_{7}$-tsvi that shall be ended is hindered accidentally and is modulated to subordinate direction again with the progression of $\mathrm{D}_{3}{ }^{4} / \mathrm{s}-\mathrm{s}{ }^{[2]}$. It is terminated perfectly with s-D-t in bar 15 to 16 and repeats the authentic cadence of D-t with strong strength for two times, and then the period is end. The primary chord at the last of the period turns to $\mathrm{f}$ minor of the connection as the intermediary chord.

The connection progresses from subdominant to dominant mainly, the start is the harmonic progression of $\mathrm{D}_{2}-\mathrm{t}_{6}$ of $\mathrm{f}$ minor, and in bar 21 , the main is the primary chord of $\mathrm{f}$ minor as the intermediary chord, tonality is turned to ${ }^{\mathrm{b}} \mathrm{E}$ major, which is a modulation of the second relationship. The bar 22 and 23 is the modulation sequence of bar18 and 19, and then change of tonality is ready for the entrance of chorus. 
The harmony of whole chorus follows the harmony configuration style of principal part from dominant to subdominant, the first phrase of chorus is turned to be minor to form the transformation of major and minor of tonic in the bar 29 with $\mathrm{D}_{2}$ as the intermediary chord so that the tonality of chorus contrasts with that of principal part. The second phrase of chorus terminates on the dominant seventh chord through the subdominant sustainability from bar 33 to 36 and after the authentic progression of continuous D-T to constitute and open cadence, and turns to the major of tonic --- ${ }^{\mathrm{b}} \mathrm{E}$ major with the chord as intermediary.

The scale of end is large and the harmony is rich from bar 37 to 61 . Dominant chord and subdominant chord are added from bar 40 to 41 , bar 46 to 47 and bar 49 to 50 to cause free tonality and turn to c minor of homophony at the end of bar 56 finally. The whole end can be divided into three parts: bar 37-43, 44-51 and 52-61. The end of the first two parts is complete cadence of $\mathrm{S}-\mathrm{K}_{4}{ }^{6}-\mathrm{D}_{7}-\mathrm{T}$ and $\mathrm{S}-\mathrm{D}_{7}-\mathrm{T}$, the end of the third party is half cadence of the new tune (c minor), and the end terminates on the dominant seventh chord to form an open cadence, which indicates the reappearance of principal part well. The principal part reappears completely from the bar 62 to 78 and the tonality is c minor.

\section{Development}

The development of this Sonata has an inserting-type polyphonic period structure and the features of trio, which can be divided into two parts.

The first part id from bar 79 to 107 with features of trio, and the second triad is applied much and the modulation is used from many times, and the harmony is rich. It can be divided into three paragraphs. The first paragraph can be divided into four phrases with four bars, in which, the second phrase is turned to ${ }^{\mathrm{b}} \mathrm{E}$ major from ${ }^{\mathrm{b}} \mathrm{A}$ major, and the melody of the third phrase is the repetition of ottava alta in the first phrase. The second paragraph only contains 4 bars (from bar 95 to 98 ), which has a polyphonic and imitative texture. It turns back to ${ }^{\mathrm{b}} \mathrm{A}$ major with tonality juxtaposition and the harmony terminates half on the dominant seventh chord. The third paragraph is from bar 99 to 107, the main harmony configuration is dominant chord and subdominant chord, the end is c minor to form an open cadence with $\mathrm{sii}_{5}{ }^{6}-\mathrm{DD}_{5}{ }^{6}$-D as harmonic progression so as to introduce the next part.

The second part of the development is from bar 107 to 120, the beginning tone overlaps with the tone at the end of the previous part, and the main texture is chord resolution. The harmony from bar 107 to 112 is the six repetitions from subdominant (dominant seventh chord) to dominant. It repeats the octave in bass, mediant and treble with the tow bars of bar 107 and 108 to cause tense atmosphere. The rhythm resolved by the chord from bar 113 to 115 becomes tercet, the chord-inversion upgoing sequence of the melody part progression broadens the whole range gradually, and up to bar 116, the chord resolution goes up to $\mathrm{f}^{3}$ tone. The landmark downgoing grading appears from bar 117 to 120 to form a melody line in the type of cascade. The harmony constitutes subdominant sustainability on subdominant harmony from bar 113 to 120 to prepare the entrance of recapitulation.

\section{Recapitulation and coda}

The principal part of recapitulation is the shrinking reappearance of the principal part of exposition with only the first 8 bars, and their harmony configuration is same exactly. The connection is changed and the scale is smaller much with only 5 bars, which is mainly developed with the material of the second phrase in the theme of the principal part. The sequence modulation is applied for the harmony and turns to $\mathrm{C}$ major with the small subdominant as intermediary at the end. The tonality of subdominant submits to C major, which also is the key to identify this tune as Sonata form. Similarly, the harmony progresses mainly from subdominant to dominant. The end harmony of chorus turns to the subdominant and subdominant altered chord appears (augmented triad structure), and the harmony color is novel. The end reflects the function of end well. The bars from 143 to 152 are the first paragraph of the end, which progresses through the conversion between subdominant and re-subdominant and the authentic harmony of subdominant and dominant, terminates completely with $\mathrm{S}-\mathrm{K}_{4}{ }^{6}-\mathrm{D}_{7}-\mathrm{T}$, and summarizes the recapitulation. The part form bar 154 to 170 is the second paragraph of end with a chorus texture, in which, the harmony changes greatly from $\mathrm{C}$ major to $\mathrm{C}$ minor, ${ }^{b} \mathrm{~A}$ major, $\mathrm{C}$ major, and returns to $\mathrm{c}$ minor through repeated tonality changes. The tonality 
changes in this paragraph seem to be the summary of the previous tonality changes of the composition. To bar 166, the music is not over but form subdominant sustainability for the reappearance of principal part. The part from bar 171 to 182 is the shrinking reappearance of the principal part of exposition (omit the supplement) and the harmony disposal is same completely.

The coda is from bar 183 to 210 for 28 bars with a large scale, and the tonality is on c minor. It can be divided into three paragraphs. The first paragraph is from bar 183 to 193, in which, the subdominant chord are its second chord are applied much to deviate the tonality to the subordinate direction, and the first phrase is over completely with the harmonic progression of $\mathrm{S}_{6}$-DDvii ${ }_{5}{ }^{6}-\mathrm{K}_{4}{ }^{6}$-D-t. In the second phrase, the tercet in the first phrase is repeated with octave, and then it is expanded to turn the pause into a hidden melody and expand the sequence of interval, and is over completely with the progression of sii $_{6}$-D-t. The second paragraph is from bar 194 to 202, and the harmony configuration is the complete progression of $\operatorname{sii}_{5}{ }^{6}-\mathrm{D}_{7}-\mathrm{t}_{6}$ and repetition, and then it turns to ${ }^{\mathrm{b}} \mathrm{A}$ major with Napoli sixth chord as intermediary. The part from bar 119 to 122 is the half cadence of this paragraph based on the subdominant harmony, and the melody is a line with landmark downgoing grading in the type of cascade. The third paragraph is from bar 203 to 210, in which, the harmony of the first bars is the plagal progression and repetition of ${ }^{\mathrm{b}} \mathrm{A}$, and next, it turns to c minor of homophony with $\mathrm{T}$ as intermediary, and for the melody, the line with landmark downgoing grading in the type of cascade appears for the last time, and the harmony configuration is the perfect cadence of DDvii ${ }_{5}{ }^{6}-\mathrm{K}_{4}{ }^{6}-\mathrm{D}_{7}$-t.

\section{Musical structure analysis}

The structure of the third movement of Beethoven piano Sonata Op.13, Pathetique, is rondo-Sonata, in which, reappearance of principal part is added after the chorus of the exposition and recapitulation, the middle part is the insertion constituted by the new theme, and the chorus of recapitulation forms the uniform Sonata principle of tonality. Therefore, this movement is in Sonata form and the whole Sonata is in rondo form, and it can be identified as rondo-Sonata. ${ }^{[3]}$

\section{Exposition}

Exposition (from bar 1 to 78). The principal part is from bar 1 to 17, which can be divided into phrase a containing 8 bars and phrase b containing 9 bars, both phrases are authentic cadence, and the tonality is on c minor. The connection id from bar 18 to 24 containing two phrases with modulation sequence. The four bars of the first phrase are on $\mathrm{f}$ minor, the four bas of the second phrase are on ${ }^{\mathrm{b}} \mathrm{E}$ major, and the end and chorus of the second phrase are connected with the first tone of chorus tightly to constitute immersive cadence ${ }^{[4]}$. The chorus is from bar 25 to 36, in which, the tonality of the first bar on ${ }^{b} \mathrm{E}$ is the relative major of principal part, and then it turns to ${ }^{\mathrm{b}} \mathrm{e}$ of minor of tonic, and finally it turns back to ${ }^{\mathrm{b}} \mathrm{E}$ major with dominant seventh chord as common chord. The whole can be divided into two phrases. The first phrase contains 9 bars, and the melody is graceful full of joyous singing quality. The second phrase contains 4 bars, m.s. is column chord and m.d. is tercet consistent with the rhythm of the end. The end is from bar 37 to 61 , the tonality of the first half is ${ }^{b} \mathrm{E}$ major, and the second half turns to c minor for the reappearance of the principal part. This part can be divided into three phrases: the first phrase is from bar 37 to 43 taking the chord resolution of tercet of m.s. and m.d. as the principal; the second phrase is from bar 4 to 51 mainly in chorus style with polyphony factor, which seemingly indicates the style of insertion; the third phrase is from bar 51 to 61, which also takes the chord resolution of tercet of m.s. and m.d. as the principal, and finally the melody goes down with cascade to prolong this part freely on the dominant seventh chord, waiting the reappearance of principal part with rondo features. The part from bar 62 to 78 is the complete reappearance of principal part.

\section{Development}

The development (from bar 79 to 120) is in insertion type. Its lengthwise part structure is similar to that of trio. The core theme is from bar 79 to 82 containing two parts with singing quality. During the bar from 83 to 86, the technique of echo and imitation between treble and bass is applied; the bars 
from 87 to 94 is trio based on the previous 8 bars with the development techniques such as the syncopation formed by part exchange and Canon imitation; during the four bars in the middle (from bar 95 to 98), a short imitation structure appears. Bar 99 starts to use the upgoing and downgoing melody of the scale of quaver staccato to be at the bass and then turn to treble, and terminate at the subdominant chord to form half cadence. The bars from 107 to 116 are the resolution melody of subdominant chord in the pattern of alternate echo of m.s. and m.d. and the primary chord, which has sense of flowing. The final part is still the landmark downgoing melody in the type of cascade so that this part can be prolonged freely on the dominant seventh chord and the recapitulation appears urgently.

\section{Recapitulation and coda}

Recapitulation (from bar 121 to 182). The principal part of the first bars has no change, and the connection is from bar 129 to 130, with the appearance of $\mathrm{f}$ minor from sequence modulation to subdominant. The tonality of the chorus (from bar 134 to 143) turns to C major, which is the same as that of homophony and reflects the unity principle of the tonality in Sonata form. The breathing clause of the melody is obvious. The end (from bar 144 to 170) is the modulation reappearance of the end in the exposition, and changes appear at the end similar to subdominant sustainability preparing for the final successive reappearance of the principal part. The reappearance of the principal part (from bar 171 to 182) is the shrinking reappearance of the principal part in the exposition with more sense of ending.

Coda (from bar 183 to 210). The beginning is overlapped melody, new material appears behind, and next, the landmark downgoing melody in the type of cascade after the progressing melody of a string of tercet and the downgoing fine material of the fifth grading through turning Napoli sixth chord into ${ }^{\mathrm{b}} \mathrm{A}$ major to make this part temporarily pause on the dominant seventh chord extended freely. The core theme of $\mathrm{p}$ turns to c minor through repeated octave, and after the weaker guiding dominant seventh chord and cadence of sixth-four chord, the landmark melody of downgoing tercet in the type of cascade appears again and the whole Sonata is over perfectly on the primary chord of homophony (c minor).

\section{Conclusion}

By the analysis of above, we can see that the harmony configuration in the third movement shows romance in the classical, the tonality distribution is homophony mainly, but also contains modulation and transposition, showing a free mood. The theme of principal part runs over with young joy and irresistible vitality, and the application of subordinate triad in the seemingly lively melody changes the color of harmony progressively. The subordinate theme in major is sprightly, but it hints unstable emotion with rapid movement. The insertion expresses real doughtiness and stable will with a proclamatory firm intonation. The whole Sonata makes people appreciate Beethoven's unique thought and belief and incomparably rakish creation style. In the musical structure, it follows both the principle of Sonata form and the features of rondo form, so that the whole structure presents the structural features of rondo-Sonata.

\section{References}

[1] Beethoven, Piano Sonatas, Beijing: People's Music Publishing House, 2003.

[2] Gao Weijie, Chen Danbu, Basic Course of Musical Form Analysis. Beijing: Higher Education Press, the 2nd Edition in May, 2006.

[3] (Hungary) Leó Weiner, Forms of Instrumental Music, translated by Zhang Rui, Beijing: Beijing Music Publishing House, the 1st Edition in August, 2002.

[4] Xu Zhaoqing, the Artistic Creation and Playing Technique of Beethoven Piano Sonata Pathetique Journal of Jiangsu Institute of Education (Social Science Edition), 2009, (04). 
[5] Wang Senlin, Analysis and Playing of Third Movement of Beethoven Piano Sonata Op.13, Pathetique, Yellow River of the Song, 2010, (22).

[6] Gong Yinan, Musical Analysis and Playing Hints of the Third Movement of Pathetique, Music Space, 2015, (02).

[7] Li Qian, Zhang Ran, on the Beethoven Sonata Pathetique and Its Playing, Journal of Tai'an Institute of Education, 2008, (04).

[8] Liu Yanzhong, How to Control the Technique and Emotion in the Third Movement of Pathetique created by Beethoven, Songs Bimonthly, 2009, (02). 